RELACult - Revista Latino-Americana de Estudos em Cultura e Sociedade e-ISSN 2016/Atual: 2525-7870 | e-ISSN 2015/2016: 2447-018X

\title{
Presença feminina no Museu de Arte Leopoldo Gotuzzo
}

\author{
Presencia feminina em el Museo de Arte Leopoldo Gotuzzo \\ Female presence at the Museum of Art Leopoldo Gotuzzo
}

\begin{abstract}
Fernanda Nunes Carrara; Noemi de Campos Bretas; Vitória Valério Machado; Luiza Prates do Santos; Daniel Rodrigues Moura ${ }^{1}$
\end{abstract}

\begin{abstract}
Resumo
Esta pesquisa tem por objetivo evidenciar a importância da realização de projetos em conjunto com a comunidade, acadêmicos, professores e servidores atuantes no Museu de Arte Leopoldo Gotuzzo (MALG), Pelotas/RS, visando maior acesso ao acervo do Museu ao mesmo tempo em que são discutidas questões relacionadas a visibilidade, representatividade e empoderamento feminino no contexto artístico latinoamericano. O texto destaca as ações realizadas em decorrência do Dia do Patrimônio que teve como temática central "Ocupação Feminina". Como parte do evento foram realizadas mediações chamando atenção para as obras e a trajetória de artistas mulheres presentes na exposição "As 7 Coleções do Acervo do MALG". Serão destacadas a brasileira Maria Lidia Magliani e a paraguaia Edith Jimenez artistas de relevância na América Latina.
\end{abstract}

Palavras-chave: Edith Jimenez; Maria Lidia Magliani; Mulheres Artistas; Museu de Arte Leopoldo Gotuzzo.

\section{Resumen}

Esta investigación tiene como objetivo poner de relieve la importancia de llevar a cabo proyectos conjuntos con la comunidad, académicos, profesores y servidores activos en el Museo de Arte Leopoldo Gotuzzo ( MALG ), Pelotas / RS, en busca de un mayor acceso a la colección del museo, al mismo tiempo temas se discuten en relación con la visibilidad e la representación de la mujer en el contexto artístico latinoamericano. El texto destaca las medidas adoptadas como consecuencia de Día del Patrimonio, que tuvo como tema central "La ocupación femenina". Como parte del evento se llevaron a cabo mediaciones que llaman la atención sobre las obras y la historia de las mujeres artistas en la exposición " As 7 coleções do acervo do Malg". Ellos serán resaltados brasileña Maria Lidia Magliani y paraguay Edith Jimenez artistas relevantes en América Latina.

Palabras Claves: Edith Jimenez; Maria Lidia Magliani; mujeres artistas; Museu de Arte Leopoldo Gotuzzo.

\begin{abstract}
This research aims to highlight the importance of carrying out joint projects with the community, academics, teachers and active servers in the Museum of Art Leopoldo Gotuzzo (MALG), Pelotas / RS, seeking greater access to the Museum's collection at the same time issues are discussed related to visibility, representation and women's empowerment in the Latin American artistic context. The text highlights the actions taken as a result of Heritage Day, which had as its central theme "Women Occupation". As part of the event were held mediations calling attention to the works and the history of women artists in the exhibition "The 7 Collections of the Acquis of MALG". They will be highlighted Brazilian Maria Lidia Magliani and Paraguayan Edith Jimenez relevant artists in Latin America.
\end{abstract}

Keywords: Edith Jimenez; Maria Lidia Magliani; Museum of Art Leopoldo Gotuzzo; women artists.

\footnotetext{
${ }^{1}$ Graduandos em Artes Visuais; Universidade Federal de Pelotas - UFPel; Pelotas, Rio Grande do Sul, Brasil; dharafernanda.nunes@hotmail.com.
} 


\section{Introdução}

O Museu de Arte Leopoldo Gotuzzo (MALG), ligado ao Centro de Artes da Universidade Federal de Pelotas, completa em 2016 trinta anos de existência. Dentre as comemorações organizadas, destacamos a participação do Museu na programação do Dia do Patrimônio. As atividades ocorridas entre 19 e 21 de agosto na cidade de Pelotas, tiveram como proposta articuladora das diversas ações, temáticas relacionadas ao eixo "Ocupação Feminina". O Dia do Patrimônio, em sua quarta edição, buscou a promoção do patrimônio arquitetônico da cidade através de visitações guiadas aos prédios do Centro Histórico da cidade e outras atividades. Em tal contexto, para além das explicações a respeito do prédio no qual o Museu está situado atualmente, destacou-se a presença de artistas mulheres latinoamericanas na exposição intitulada "As 7 coleções do acervo do MALG”.

O presente artigo tem como finalidade destacar o importante papel de mulheres como a professora Luciana Renck Reis, idealizadora e fundadora do MALG no ano de 1986, da artista brasileira Maria Lidia Maglianni e da paraguaia Edith Jimenez, ambas representadas na exposição mencionada anteriormente. Destacamos também a relevância do Museu de Arte Leopoldo Gotuzzo, único museu de arte da cidade de Pelotas e o reconhecimento recebido pelo Dia do Patrimônio, conjunto de atividades promovidas pela prefeitura de Pelotas, por meio da 29a Edição do Prêmio Rodrigo Melo Franco de Andrade, do Instituto do Patrimônio Histórico e Artístico Nacional (Iphan).

Ao articular a proposta do Dia do Patrimônio com a exposição em cartaz no Museu evidenciamos a presença de trabalhos realizados por artistas mulheres. A exposição "As 7 coleções do Acervo do MALG” foi organizada a partir de obras que compõe o acervo do museu. Das sete coleções presentes na mostra, quatro foram escolhidas para salientar a trajetória artística e pessoal de mulheres artistas. As artistas em destaque pela coleção L.C. Vinholes foram Isabel Pons e Edith Jiménez selecionadas pela inovação das técnicas utilizadas em seu trabalho como o uso de cor na gravura; na coleção Século XX foi ressaltada a artista Maria Lídia Magliane que em suas obras retrata uma forte influência do movimento feminista; já na coleção Século XXI, a representante foi Lenir de Miranda, artista conceitual conhecida por obras que carregam alto grau de narratividade e por fim, na coleção Belas Artes evidenciam-se duas mulheres, sendo elas Hilda Mattos, aluna da primeira turma de Artes da EBA (Escola de Belas Artes em Pelotas-RS) e Carlinda Valente, professora fundadora da disciplina de Gravura na EBA. 


\section{Desenvolvimento e Discussão}

A presença das mulheres no meio artístico durante muito tempo teve pouca visibilidade. As articulações surgidas entre o âmbito acadêmico e o movimento feminista a partir da década de 1960 fizeram com que o cenário começasse a sofrer modificações. Segundo Neide Jallageas (XXXX, p. 03)

\footnotetext{
Ainda que a figura da mulher tenha sido tema de praticamente a totalidade das artes visuais e sendo assim o objeto do olhar tanto do autor ou autora quanto do espectador ou espectadora - e de inúmeros estudos sobre a sua "imagem", é recente a atenção que começa a ser dirigida à mulher artista, à mulher sujeito da ação de desenhar, pintar, esculpir, gravar, fotografar.
}

Embora a presença feminina tenha crescido em diversos âmbitos ainda se faz necessário destacar a trajetória de certas mulheres para que as mesmas não sejam esquecidas. No caso tanto da fundação do Museu de Arte Leopoldo Gotuzzo quanto da Escola de Belas Artes, de fundação anterior ao Museu, articulações e insistências por parte de duas mulheres tiveram papel fundamental: Marina de Moraes Pires e Luciana Renck Reis.

A professora de artes no Instituto de Educação Assis Brasil, Marina de Moraes Pires, no final da década de 1940 lutava em busca da concretização da Escola de Belas Artes para possibilitar o aprofundamento do conhecimento artístico em Pelotas, porém, somente com a presença masculina de Aldo Locatelli que os pelotenses decidiram dar credibilidade e investir neste projeto. Assim, foi criada a EBA em 1949(ROCHA, Consuelo, 2010).

Em 1972, a escola passou a funcionar como unidade de ensino das artes plásticas e posteriormente tornou-se parte da Universidade Federal de Pelotas. Neste período, seu patrimônio artístico passou para o imóvel que servia de sede da Reitoria. A EBA se transformou em ILA (Instituto de Letras e Artes, atual Centro de Artes), e a responsabilidade do acervo passou a ser da faculdade. Inicialmente esse acervo contava com três grandes coleções: obras de autoria de Leopoldo Gotuzzo, doação feita por Bertilde Trápaga Simões de coleções particulares e Dr. João Gomes de Mello Filho. Em 1983 iniciou-se um projeto de conservação e restauro dessas coleções comandado por Luciana Renck Reis em parceria com a também professora do departamento de Artes Visuais do ILA, Yeda Machado Luz, o que posteriormente levou à elaboração do projeto da Pinacoteca.

Durante a idealização do Museu, Luciana Renck Reis foi até o Rio de Janeiro onde residia Leopoldo Gotuzzo e convidou-o para tornar-se o patrono do Museu solicitando que o Museu pudesse levar seu nome. Seu convite é aceito e a permissão lhe é concedida, deste modo o Museu de Arte Leopoldo Gotuzzo surgiu (ROCHA, Consuelo, 2010). 
RELACult - Revista Latino-Americana de Estudos em Cultura e Sociedade

Revista Latinoamericana de Estudios en Cultura y Sociedad | Latin American Journal of Studies in Culture and Society V. 02, Ed. Especial, dezembro, 2016, p. 340-348| periodicos.claec.org e-ISSN 2016/Atual: 2525-7870 | e-ISSN 2015/2016: 2447-018X

Em homenagem às grandes mulheres que lutaram por sua criação e depois pela permanência do MALG, o Museu tem os nomes de Marina de Moraes Pires e Luciana Renck Reis intitulando suas duas primeiras salas expositivas.

A exposição "As 7 Coleções do Acervo do MALG" contou com a presença de inúmeras artistas, citadas anteriormente, dentre elas destacam-se as latino-americanas, Edith Jiménez (Figura 1), natural de Asunción no Paraguai, pintora e gravurista (uma das pioneiras á utilizar cor na gravura) e Maria Lídia Magliani (Figura 2), a primeira artista negra a se formar na UFRGS (Universidade Federal do Rio Grande do Sul) e grande atuante no movimento feminista.

O destaque dado às obras de artistas mulheres durante roteiros realizados junto ao público teve como objetivo destacar a produção feminina presente no acervo do museu e discutir a importância dessas mulheres na história da instituição e seu papel relevante na própria história da arte latino americana.

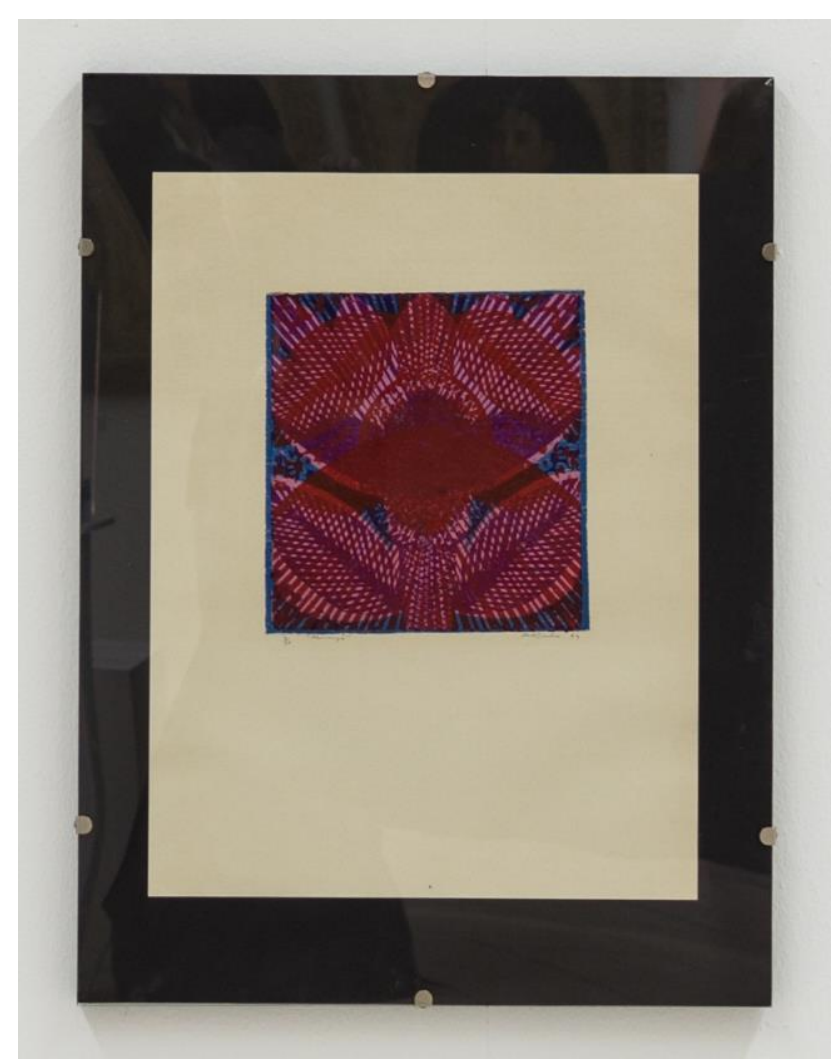

Figura 1 - Imagem da Obra de Edith Jiménez

Fonte: Daniel Rodrigues Moura 

e-ISSN 2016/Atual: 2525-7870 | e-ISSN 2015/2016: 2447-018X

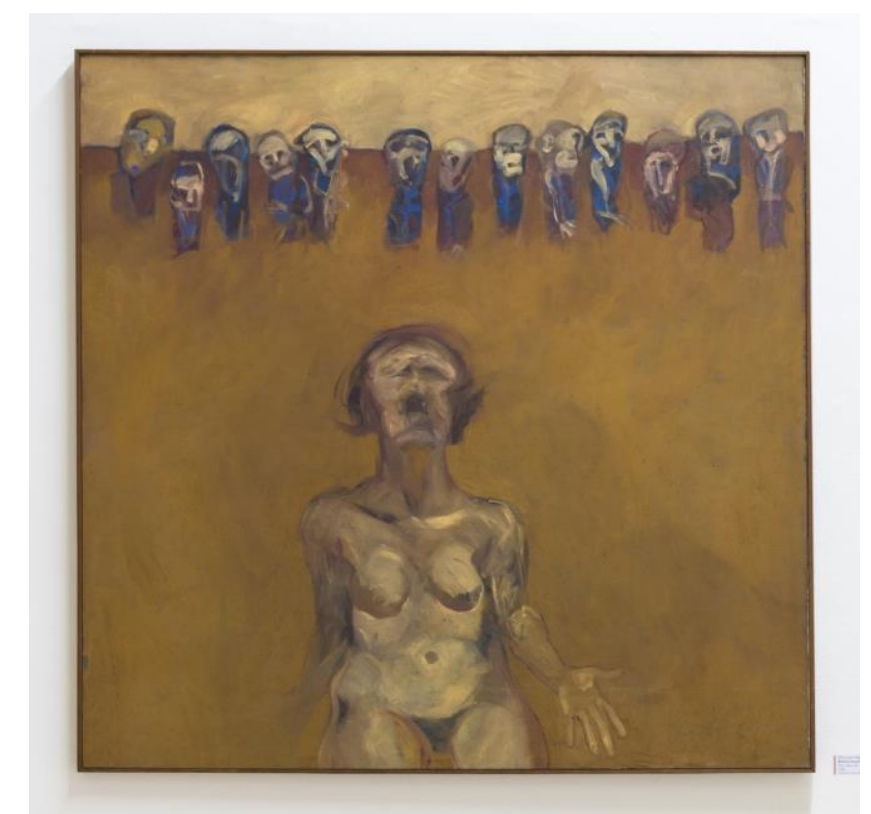

Figura 2 - Imagem da Obra de Maria Lídia Magliani Fonte: Daniel Rodrigues Moura

Além das imagens já mostradas pertencentes ao Museu de Arte Leopoldo Gotuzzo (MALG), a pesquisa estendeu-se à biografia e aprofundamento sobre o processo artístico das referidas artistas.

\section{Edith Jiménez}

Edith Jiménez foi um ícone na arte paraguaia no século XX. Iniciou seu estudo na área de pintura em 1943, sendo orientada por Jaime Bestard, como o qual desenvolveu um trabalho que possuía traços pós-impressionistas. No fim da década de 50 iniciou seus estudos em gravura, principalmente xilogravura, onde dedica-se por aproximadamente 10 anos.

Em 1962 apresenta seus primeiros trabalhos em gravura (xilogravura) no Brasil, contendo forte característica expressionista abstrata e temas cósmicos como por exemplo, lua, céu, paisagem, entre outros, todos provenientes da natureza do cosmo.

Foi condecorada no Brasil em 1997 após a sua trajetória ao longo de suas exposições e sua participação na Bienal de São Paulo em 1975. 
RELACult - Revista Latino-Americana de Estudos em Cultura e Sociedade

Revista Latinoamericana de Estudios en Cultura y Sociedad | Latin American Journal of Studies in Culture and Society V. 02, Ed. Especial, dezembro, 2016, p. 340-348 | periodicos.claec.org e-ISSN 2016/Atual: 2525-7870 | e-ISSN 2015/2016: 2447-018X

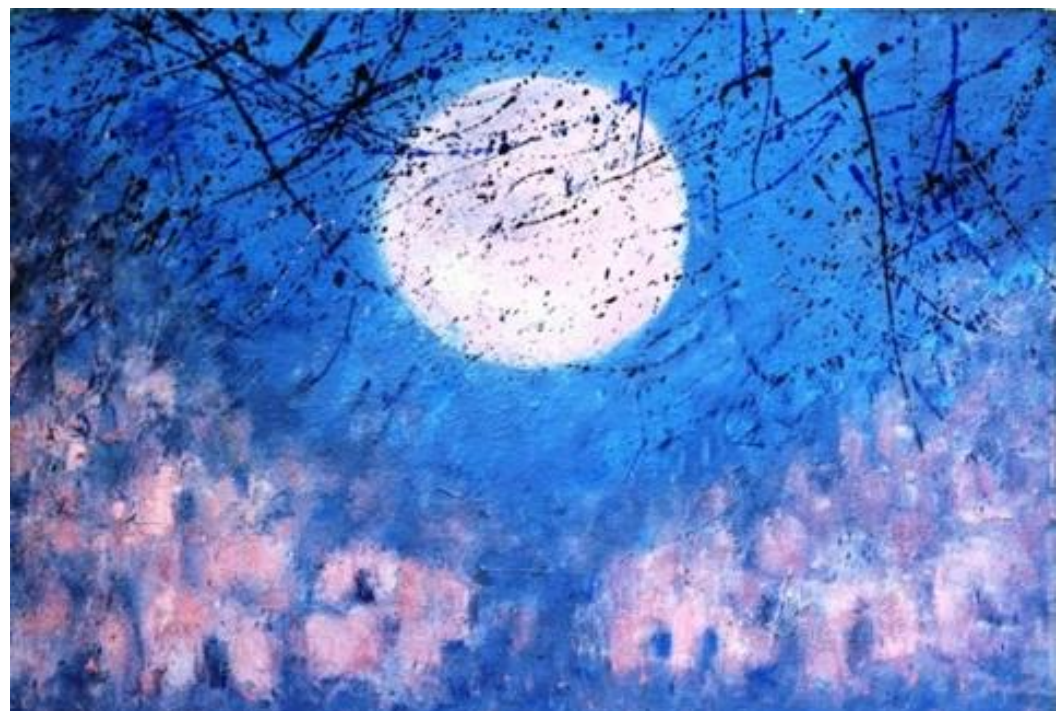

Figura 3 - Imagem da Obra de Edith Jiménez

Fonte: Portal Guarani

Acima, a Imagem "Sem Título" de Edith retrata a paisagem de um luar onde prevalece cores fortes como o azul e tons de magenta e demonstra as pinceladas expressionistas abstratas já citadas.

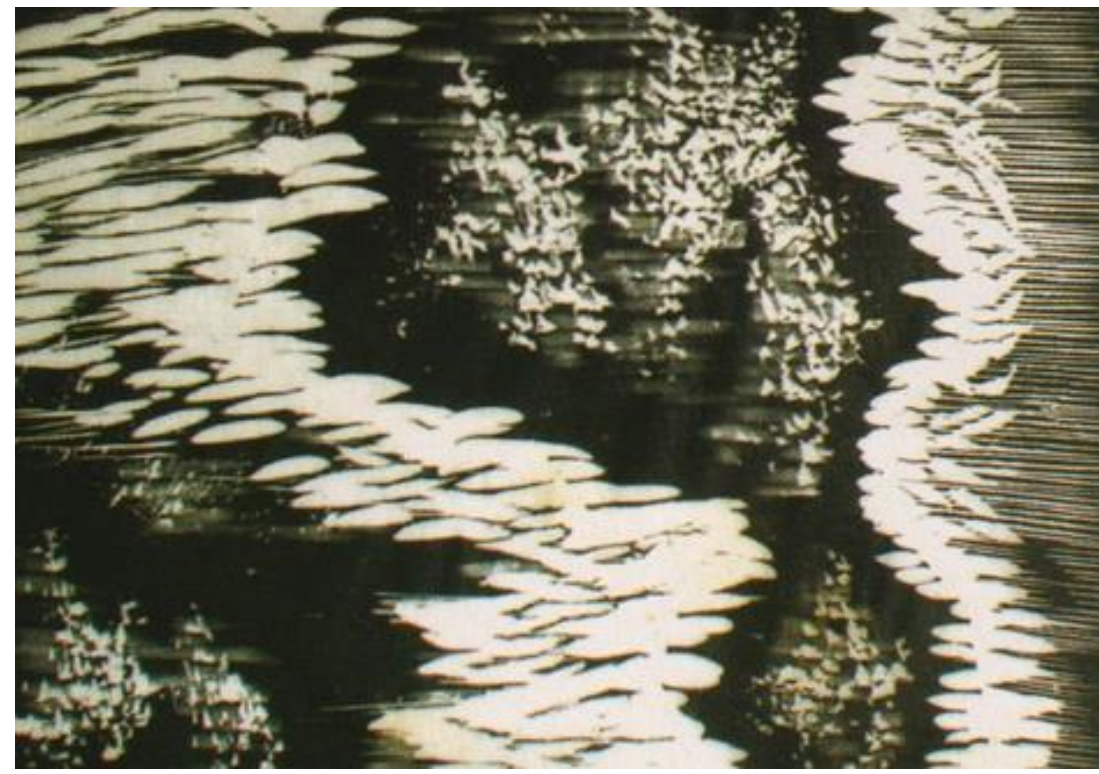

Figura 4 - Imagem da Obra de Edith Jiménez

Fonte: Portal Guarani

A gravura referente à Figura 4 tem como tema o abstracionismo e as características mais marcantes da técnica utilizada e aprimorada pela artista. Como a linguagem da gravura foi a mais explorada, a artista desenvolveu esta técnica em nível superior comparado as demais. 
RELACult - Revista Latino-Americana de Estudos em Cultura e Sociedade

Revista Latinoamericana de Estudios en Cultura y Sociedad | Latin American Journal of Studies in Culture and Society V. 02, Ed. Especial, dezembro, 2016, p. 340-348| periodicos.claec.org e-ISSN 2016/Atual: 2525-7870 | e-ISSN 2015/2016: 2447-018X

\section{Maria Lídia Magliani}

Maria Lídia Magliani, pintora e desenhista pelotense é uma das artistas gaúchas de maior destaque devido á seus engajamentos feminista (Figura 5) e político (Figura 6), este principalmente na época da Ditadura Militar no Brasil, onde estampou com seus desenhos as páginas dos jornais da época de 70 denunciando os acontecimentos violentos.

Também trabalhou como ilustradora desenvolvendo trabalhos para Folha da Manhã, Zero Hora e O Estado de São Paulo, além de ter criado cartazes e capas de livros.

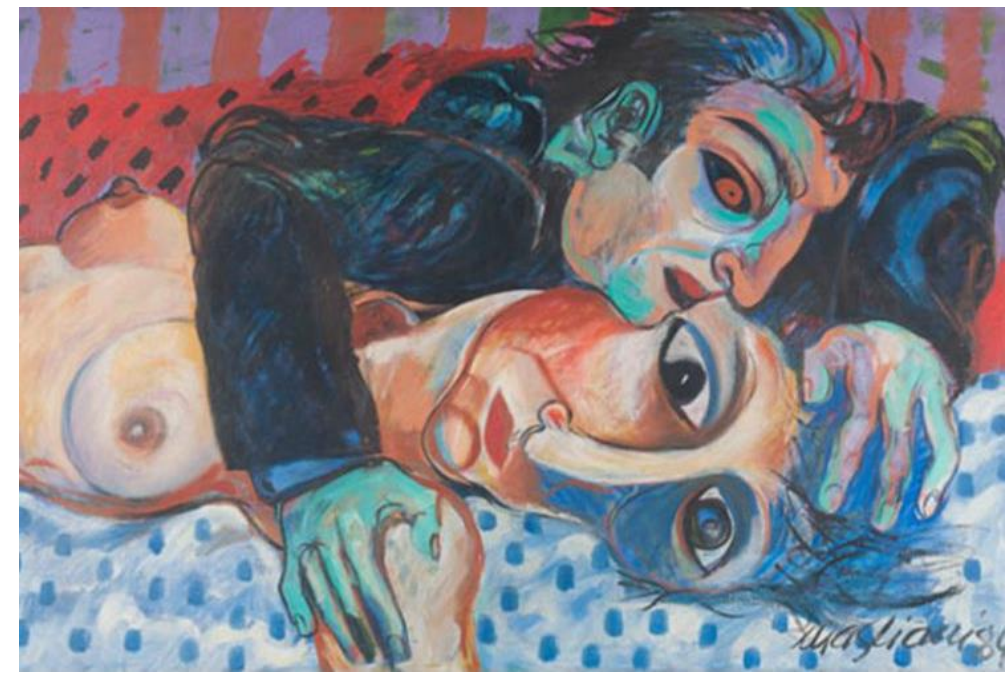

Figura 5 - Imagem da Obra de Maria Lídia Magliani

Fonte: Acervo SMC/Divulgação/JC

A imagem acima retrata um dos temas mais aprofundados pela artista, o feminismo, trazendo a discussão sobre o abuso com as mulheres.

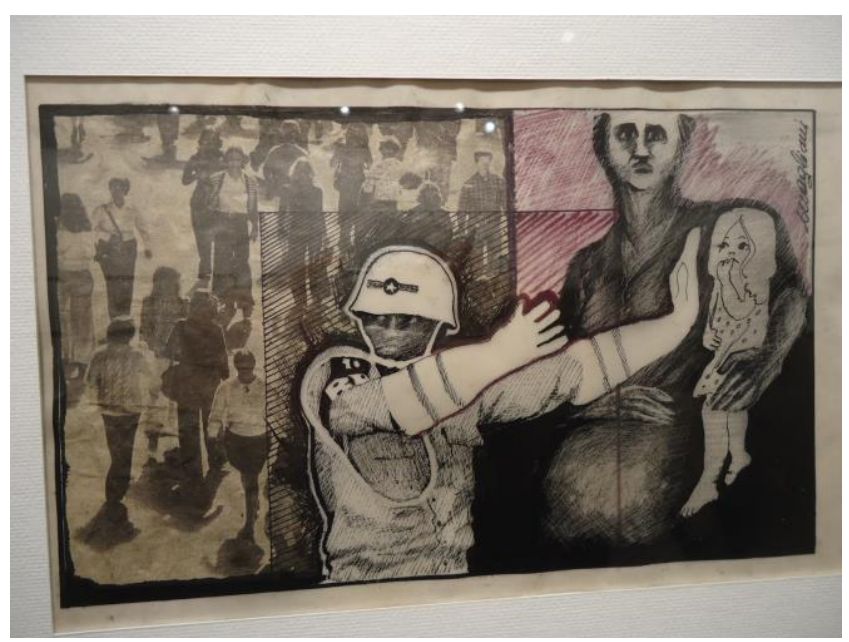

Figura 6 - Imagem da Obra de Maria Lídia Magliani

Fonte: PET Interdisciplinar Feevale 

e-ISSN 2016/Atual: 2525-7870 | e-ISSN 2015/2016: 2447-018X

A Figura 6 descreve uma situação recorrente durante a Ditadura Militar no Brasil, onde representa o abuso de poder, as privações e a imposição do respeito através do medo.

\section{Conclusões}

O presente trabalho apresenta uma série de relações entre as lutas feministas em um contexto global e as articulações realizadas por mulheres na cidade de Pelotas para a criação de uma Escola de Belas Artes e depois de um Museu de Arte. Também se faz importante, através de ações educativas realizadas a partir das mostras realizadas no Museu de Arte Leopoldo Gotuzzo destacar a presença e a representatividade de artistas mulheres no cenário artístico, possibilitando assim reflexões sobre temas contemporâneos de extrema importância como representatividade e empoderamento feminino.

\section{Referências}

AVILA, Kathleen; ANGELI, Juliana; BONILHA, Caroline. Ação educativa MALG: Museu, Escola, Comunidade. IN: MICHELON, Francisca Ferreira; NUNES, João Fernando Igansi; BUSSOLETTI, Denise Marcos (org). Anais do Congresso de Extensão e Cultura da UFPel. Pelotas: Ed. da UFPel, 2015.

ROCHA, Maria Consuelo Sinotti. Museu de Arte Leopoldo Gotuzzo: contribuição e integração com o ensino de Arte através de seu Setor Educacional. Universidade Federal de Pelotas, Trabalho de Conclusão de Curso, 2010. Disponível em: $<$ http://wp.ufpel.edu.br/especializacaoemartesvisuais/files/2013/06/Maria-Consuelo-SinottiRocha---2010.pdf >. Acesso em: 26 agosto de 2016.

JALLAGEAS, Neide. Respostas mínimas a questões máximas: sobre a (in)visibilidade da mulher artista na história da arte. Disponível em: www.miniweb.com.br/artes/artigos/gt15a1.pdf. Acesso em: 27 de agosto de 2016. 